\title{
Prototype formation: Central tendency model vs. attribute-frequency model
}

\author{
ROBERT L. SOLSO and JUDITH E. McCARTHY \\ University of Idaho, Moscow, Idaho 83843
}

\begin{abstract}
In this experiment, subjects were shown a series of three-digit numbers. Following this stage, the subjects were shown some old items, some new items, a prototype based on frequently repeated digits, and a prototype based on the mean of the group. Subjects erroneously identified the frequency-related, but not the mean, prototype as an old item. Support was found for the attribute-frequency model of prototypes.
\end{abstract}

Recent findings reported in the psychological literature leave little doubt that information derived from experience with exemplars is commonly stored in terms of a prototype or summary representative (see Bartlett, 1932; Franks \& Bransford, 1971; Medin \& Schaffer, 1978; Neumann, 1977; Posner \& Keele, 1968; Reed, 1972; Solso \& Raynis, 1979). There is, however, considerable debate as to which model of human memory best represents data collected in prototype experiments.

One model holds that a prototype represents the mean or central tendency of the set of exemplars; the other holds that the prototype represents the mode of the set of exemplars, usually the result of a combination of high-frequency attributes. An example of a central tendency model was developed by Posner and Keele (1968). According to this model, a prototype is represented mathematically by a hypothetical point in multidimensional space at which the means of the distances along all attributes intersect. Thus, a prototype is an abstraction stored in memory that represents the central tendency of each category. The prototype is altered by each experience with a new set member, with the influence of individual stimuli decreasing as the total number of experienced set members increases. This model is also described by Reed (1978), who suggests that features can be given more weight in the computation of the mean distance as a function of their ability to discriminate between categories. An assumption of the central tendency model is that both the prototype and its features need not be directly experienced, since the prototype and its components are the products of an averaging process based on direct experience with exemplars.

An example of a frequency model (or independent cue model; Medin \& Schaffer, 1979) is Neumann's $(1974,1977)$ model, in which a prototype or best example is the pattern that incorporates the most frequently experienced feature within each attribute. While the prototype is often unique, because it may

Reprint requests should be addressed to R. Solso, Department of Psychology, University of Idaho, Moscow, Idaho 83843. consist of a new combination of attributes, the features themselves have been experienced contrary to a central tendency model. Neumann (1977) experimentally compared the two types of prototype models and found that, to some extent, characteristics of the stimuli dictate which process a subject will use to formulate a prototype.

The cognitive mechanisms postulated by one model are distinctly different from the mechanisms postulated by the other. In the present report, we hypothesize that both forms of prototype representations could take place simultaneously in human memory. In order to test this hypothesis, we developed numerical exemplars composed of three digits and asked subjects to judge their recognition of two types of "prototypes": one based on the frequency of components and another based on the arithmetic average of the series.

\section{METHOD}

\section{Subjects}

Subjects were 22 students from an experimental design course at the University of Idaho who volunteered for participation in partial fulfillment of course requirements.

\section{Stimuli}

Fifteen three-digit numbers were developed and served as exemplar stimuli. The three-digit series were composed in such a way that a specific number did not appear more than once in any of the three positions with the exception of the $\mathbf{P}_{\mathbf{f}}$ (prototype based on frequency).

In the current example, the number 832 was the $P_{f}$. The digits 8,3 , and 2 each appeared five times in the first, second, and third positions, respectively. Additional constraints were that the digit 0 could not appear in the first position, no repeated digits were allowed, and noticeable sequences were avoided (e.g., 123). Each digit appeared only once per position, except in four cases distributed over the three positions in which a digit appeared twice and in the case of 8,3 , and 2 . From this exemplar series, an arithmetic average was calculated to be 576. This number was called $\mathrm{P}_{\overline{\mathrm{X}}}$ (prototype based on the mean).

Recognition stimuli consisted of 12 three-digit numbers. Five numbers were selected and ordered randomly from the exemplar series. Five new numbers were composed with minimum commonality to the $\mathbf{P}_{\mathbf{f}}$ and the $\mathrm{P}_{\overline{\mathbf{X}}}$. The $\mathbf{P}_{\mathbf{f}}$ and the $\mathrm{P}_{\overline{\mathbf{X}}}$ were also used. The $P_{f}$ and $P_{\bar{X}}$ did not appear in the first or last position. 


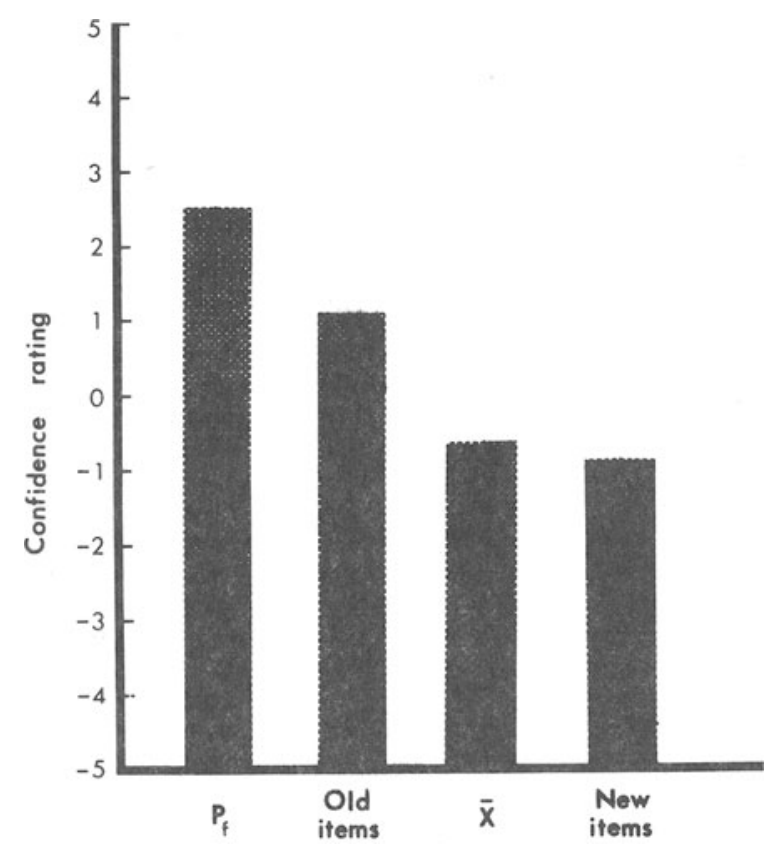

Figure 1. Average confidence ratings for $P_{f}$, old items, $\mathbf{P} \overline{\mathbf{X}}$, and new items.

\section{Procedure}

Subjects, who were run in one group, were instructed that a series of numbers would be shown and that they were to memorize them. The 15 exemplar digits were projected on a screen by means of an overhead projector. Each item was shown for $5 \mathrm{sec}$ with about a 2 -sec interitem interval. The subjects were then asked to write the names of states in alphabetic order. Following $5 \mathrm{~min}$ of this task, a recognition test was administered. Each item of the recognition stimuli was shown for about $7 \mathrm{sec}$ with a 2-sec interitem interval. Subjects were asked to decide whether or not the three-digit number was a member of the original series. Following this decision, they were asked to rate their choice on a graduated confidence scale that ranged from 1 (unsure) to 5 (very confident).

\section{Results}

The data were summarized and classified into old items, new items, $P_{f}$, and $P_{\bar{X}}$. The means of these four groups are shown in Figure 1. A one-way analysis of variance yielded $F(3,260)=10.04(p<.001)$. In order to make specific comparisons among the groups, a protected least-significant difference (1.s.d.) test for proportional means was calculated, with the following results: $P_{f}$ vs. $P_{\bar{X}}, t(42)=3.47(p<.001)$; $P_{f}$ vs. new items, $\mathrm{t}(130)=4.50(\mathrm{p}<.001) ; \mathrm{P}_{\mathrm{f}}$ vs. old items, $\mathrm{t}(130)$ $=1.70(\mathrm{p}<.10)$; new items vs. $P_{\bar{X}}, t(130)=2.09$ $(\mathrm{p}<.05)$; and old items vs. new items, $\mathrm{t}(218)=4.23$, $(\mathrm{p}<.001)$. $^{1}$

\section{DISCUSSION}

In the present experiment, we hypothesized that a prototype based on a frequency model and on a central tendency model could develop simultaneously. Our data conclusively support a frequency model. Using numerical exemplars of the sort described in this report, there is little doubt that subjects formed a $\mathbf{P}_{\mathbf{f}}$ based on the sum of frequently experienced positive digits.
All subjects in our sample falsely identified the $P_{f}$ as an old item. Although the confidence ratings of the $P_{f}$ were not reliably higher than those of the old items (mean 2.54 vs. mean $=1.14$ ), there appeared to be a tendency for subjects to evaluate the $\mathbf{P}_{\mathbf{f}}$ as an old item with greater confidence than they evaluated the actual old items. Such a tendency is consistent with earlier work (Homa \& Vosburg, 1976; Solso \& McCarthy, Note 1) in which a prototype was regarded as an "epitome of a pattern" or the "best exemplar." A frequency model of prototype formation seems to account for these results.

We found little evidence that a central tendency model would account for our results. The central tendency was defined in this experiment as a point in multidimensional space at which the means of attributes intersect. That value was assumed to be the mean of the entire series $\left(P_{\bar{X}}\right)$. One may argue that the mean of the entire series is an inappropriate index of the $P_{\bar{X}}$ and that the proper $\mathrm{P}_{\overline{\mathrm{X}}}$ value should correspond to the mean of each of the three positions. That speculation awaits further validation and was not testable in the present experiment.

Our lack of support for the central tendency model does not mean that the model should be abandoned: Such a consideration would amount to accepting the null hypothesis. We would, however, argue that, given the nature of digits as exemplars, the frequency model seems to account for the results better than the central tendency model does and that we did not find evidence for two kinds of prototypes developing simultaneously.

\section{REFERENCE NOTE}

1. Solso, R. L., \& McCarthy, J. Prototype formation of faces. Manuscript submitted for publication, 1979.

\section{REFERENCES}

Bartlett, F. C. Remembering. Cambridge: Cambridge University, 1932.

Franks, J. J., \& Bransford, J. D. Abstraction of visual patterns. Journal of Experimental Psychology, 1971, 90, 65-74.

Homa, D., \& Vosburg, R. Category breadth and the abstraction of prototypical information. Journal of Experimental Psychology: Human Learning and Memory, 1976, 2, 322-330.

Medin, D. L., \& Schaffer, M. M. Context theory of classification learning. Psychological Review, 1978, 85, 208-238.

NeumanN, P. G. An attribute frequency model for the abstraction of prototypes. Memory \& Cognition, 1974, 2, 241-248.

NeumanN, P. G. Visual prototype formation with discontinuous representations of dimensions of variability. Memory \& Cognition, 1977, 5, 187-197.

Posner, M. I., \& Keele, S. W. On the genesis of abstract ideas. Journal of Experimental Psychology, 1968, 77, 353-363.

REED, S. K. Pattern recognition and categorization. Cognitive Psychology, 1972, 3, 382-407.

REED, S. K. Category vs. item learning: Implications for categorization models. Memory \& Cognition, 1978, 6, 612-621.

Solso, R. L., \& RAYNis, S. A. Prototype formation from imaged, kinesthetically, and visually presented geometric figures. Journal of Experimental Psychology: Human Perception and Performance, 1979, 5, 701-712.

\section{NOTE}

1. It is noted that the above results have been obtained several times with different subjects and using different digits. The data from these other experiments are not reported, as they were used as a class demonstration and lack the precision of the present experiment.

(Received for publication December 15, 1980.) 